\title{
Particle-size distribution and packing fraction of geometric random packings
}

\author{
H. J. H. Brouwers \\ Faculty of Engineering Technology, University of Twente, P.O. Box 217, 7500 AE Enschede, The Netherlands
}

(Received 6 March 2006; published 26 September 2006)

\begin{abstract}
This paper addresses the geometric random packing and void fraction of polydisperse particles. It is demonstrated that the bimodal packing can be transformed into a continuous particle-size distribution of the power law type. It follows that a maximum packing fraction of particles is obtained when the exponent (distribution modulus) of the power law function is zero, which is to say, the cumulative finer fraction is a logarithmic function of the particle size. For maximum geometric packings composed of sieve fractions or of discretely sized particles, the distribution modulus is positive (typically $0<\alpha<0.37$ ). Furthermore, an original and exact expression is derived that predicts the packing fraction of the polydisperse power law packing, and which is governed by the distribution exponent, size width, mode of packing, and particle shape only. For a number of particle shapes and their packing modes (close, loose), these parameters are given. The analytical expression of the packing fraction is thoroughly compared with experiments reported in the literature, and good agreement is found.
\end{abstract}

DOI: 10.1103/PhysRevE.74.031309

PACS number(s): 45.70.-n, 81.05.Rm

\section{INTRODUCTION}

The packing of particles is relevant to physicists, biologists, and engineers. The packing fraction affects the properties of porous materials, the viscosity of particulate suspensions, and the glass-forming ability of alloys [1,2]. Furthermore, collections of hard spheres also serve as a model for the structure of simple liquids $[3,4]$. There is, therefore, practical as well as fundamental interest in understanding the relationship between the particle shape and particle-size distribution on the one hand, and packing fraction on the other. Actually, it is an old dream among particle scientists to directly relate them

The packing fraction of particles depends on their shape and method of packing: regular or irregular (random), where the latter furthermore depends on the densification. The densest packing of equal spheres is obtained for a regular (crystalline) arrangement, for instance, the simple cubic (sc), bcc, and fcc/hcp lattices, having a packing fraction of $\pi / 6(\approx 0.52), 3^{1 / 2} \pi / 8(\approx 0.68)$, and $2^{1 / 2} \pi / 6(\approx 0.74)$, respectively. Polydisperse regular packings are in development, but are difficult to describe and realize in practice [5]. The packing of binary sc, bcc, and fcc lattices is addressed by Denton and Ashcroft [6] and Jalali and Li [7]. Recently, Mahmoodi Baram et al. [8] have constructed the first three dimensional (3D) space-filling bearing.

On the other hand, in nature and technology, often a wide variety of randomlike packings are found, also referred to as disordered packings. Examples are packings of rice grains, cement, sand, medical powders, ceramic powders, fibers, and atoms in amorphous materials, which have a monosized packing fraction that depends on the method of packing [random loose packing (RLP) or random close packing (RCP)]. For RCP of uniform spheres the packing fraction $\left(f_{1}\right)$ was experimentally found to be 0.64 [9], being in line with computer generated values $[10,11]$. For RLP of spheres in the limit of zero gravity, $f_{1}=0.44$ was measured [12]. For a number of nonspherical, but regular, particle shapes the monosized packing fraction has been computed and or measured for disks [13], thin rods [14], and ellipsoids [15]. For irregular particles, much work has been done on the prediction of the unimodal void fraction using shape factors etc., but for many irregular shapes it is still recommendable to obtain the monosized void fraction from experiments.

Another complication arises when particles or atoms of different sizes are randomly packed, which is often the case for products processed from granular materials and in amorphous alloys. For continuous normal and lognormal distributions, Sohn and Moreland [16] determined experimentally the packing fraction as a function of the standard deviation. He et al. [17] reported Monte Carlo simulations of these packings. Another special class of polydisperse packings are the so-called geometric packings (i.e., the ratios of particle sizes and the ratios of pertaining quantities are constants), which are the main focus of this paper. The geometric systems can be classified in two subclassifications: (1) the packing of many discretely sized particles, and (2) the packing of continuous particle-size distributions. The packing fraction of both polydisperse particle systems depends on the particle-size distribution. The two basic theories on geometric particle packings stem from Furnas $[18,19]$ and from Andreasen and Andersen [20].

Furnas addresses in his earliest work the packing fraction of discrete two-component (binary) mixtures, which was later extended to multimodal particle packings. The packing fraction of continuously graded particles, whereby all particle sizes are present in the distribution, was studied in Ref. [20] using geometrical considerations. Based on his discrete particles packing theory, Furnas [19] also postulated a geometric rule for maximum continuous packings, i.e. the ratio between subsequent values is constant. In Sec. II hereof both theories on geometric particle packings are discussed in detail. Though attempts have been made to relate the discrete and continuous approaches of packings [21,22], a closed mathematical linking is still lacking.

In Sec. III of this paper, it is demonstrated that the multiple discrete packing theory of Furnas can be transformed to a continuously graded system with a power law distribution. It is seen that the theories on discrete and continuous pack- 


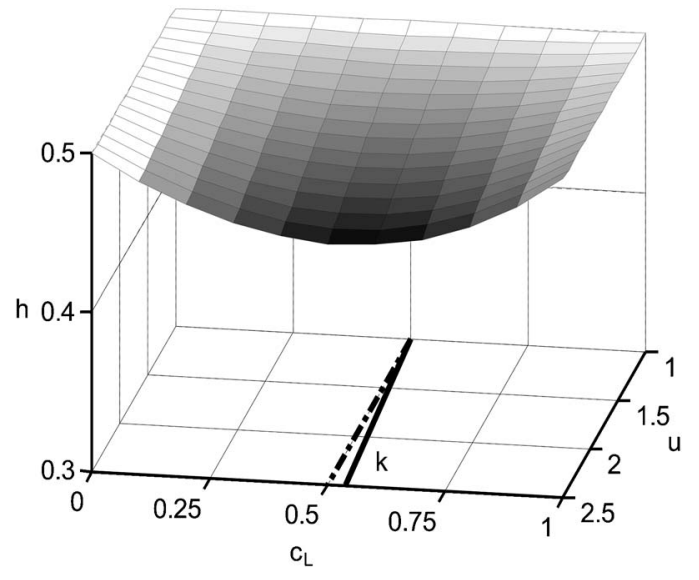

FIG. 1. Void fraction of bimodal mixes $(h)$ as a function of size ratio $d_{\mathrm{L}} / d_{\mathrm{S}}(u)$ and volume fraction of large constituent $\left(c_{\mathrm{L}}\right)$ according to Furnas [18] for $\left(1 \leqslant u \leqslant 2.5,0 \leqslant c_{\mathrm{L}} \leqslant 1\right)$, whereby the void fraction is described with a Redlich and Kister [27] type equation of the form $h\left(u, c_{\mathrm{L}}\right)=\varphi_{1}-4 \varphi_{1}\left(1-\varphi_{1}\right) \beta(u-1) c_{\mathrm{L}}(1$ $\left.-c_{\mathrm{L}}\right)\left[1+m\left(1-2 c_{\mathrm{L}}\right)\right]$, with $\varphi_{1}=0.5, \quad \beta=0.125$, and $m=-0.08(u$ $-1)^{1.7}$. The curve $\left[u, c_{\mathrm{L}}=k(u)\right]$, corresponding to $d h / d c_{\mathrm{L}}=0$ (composition of maximum packing fraction), is also included, $k(u)=0.5+\left[\left(1+3 m^{2}\right)^{1 / 2}-1\right] /(-6 m)$, as well as the symmetry line $\left[u, c_{\mathrm{L}}=1 / 2\right]$.

ings are related mathematically and are actually complementary. Next, in Sec. IV it is demonstrated that the unification of both theories also enables the prediction of the void fraction of the continuous power law packing for any particle shape. A general equation in closed form is derived that provides the void fraction as a function of distribution width $\left(d_{\max } / d_{\text {min }}\right)$, the single-sized void fraction of the particle shape considered $\left(\varphi_{1}\right)$, the distribution modulus $\alpha$, and the gradient in void fraction in the limit of monosized system to two-component system $(\beta)$. This original expression for the void/packing fraction is compared thoroughly with classical experiments reported in Ref. [20], and found to be in good accordance. It also appears that the obtained equation is compatible with an old empirical equation, first proposed in Ref. [23].

\section{DISCRETE AND CONTINUOUS GEOMETRIC PACKING OF PARTICLES}

Furnas $[18,19]$ was the first to model the maximum packing fraction of polydisperse discrete particle-size distributions, and Andreasen and Andersen [20] derived a semiempirical continuous distribution based on the insight that successive classes of particle sizes should form a geometric progression. Both theories are addressed in this section.

\section{A. Discrete bimodal packing}

Furnas [18] studied bimodal systems at first instance. By studying binary mixtures of particles, it was concluded that the greater the difference in size between the two components, the greater the decrease in void volume. From Fig. 1, a $3 \mathrm{D}$ representation of the experiments with loosely packed spheres $\left(\varphi_{1}=0.50\right)$, it can be seen that the bimodal void fraction $h$ depends on diameter ratio $u\left(d_{\mathrm{L}} / d_{\mathrm{S}}\right)$ and on the fraction of large and small constituents $c_{\mathrm{L}}$ and $c_{\mathrm{S}}$, respectively.

As illustrated by Fig. 1, Furnas [18] expressed his results in diameter ratios and volume fractions (of large and small particles). In what follows in regard to geometric polydisperse packings, it will be seen that also the ratio $r$ of large to small particles is of major relevance, here defined as

$$
r=\frac{c_{L}}{c_{S}},
$$

whereby for a bimodal packing obviously holds

$$
c_{\mathrm{S}}=1-c_{\mathrm{L}},
$$

so $r$ takes the value of 0,1 , and $\infty$ for $c_{\mathrm{L}}$ being $0,1 / 2$, and 1 , respectively.

Now let $f_{1}$ and $\varphi_{1}$ be the packing fraction and void fraction, respectively, of the uniformly sized particles, with

$$
f_{1}=1-\varphi_{1}
$$

then by combining two noninteracting size groups, one obtains as total bimodal packing and void fractions

$$
f_{2}=f_{1}+\left(1-f_{1}\right) f_{1} ; \quad \varphi_{2}=1-f_{2}=\left(1-f_{1}\right)^{2}=\varphi_{1}^{2} .
$$

This concept is applicable only when the smaller ones do not affect the packing of the larger size group. Experiments with mixtures of discrete sphere sizes $[18,24]$ revealed that this is obviously true when $u \rightarrow \infty$, but that nondisturbance is also closely approximated when $d_{\mathrm{L}} / d_{\mathrm{S}} \approx 7-10$ (designated as $\left.u_{\mathrm{b}}\right)$. For irregular particles, Caquot [23] found a comparable size ratio $\left(u_{\mathrm{b}} \approx 8-16\right)$. For such bimodal packing, the volume fractions of large $\left(c_{\mathrm{L}}=c_{1}\right)$ and small $\left(c_{\mathrm{S}}=c_{2}\right)$ size groups in the mix are

$$
\begin{aligned}
c_{\mathrm{L}}= & \frac{f_{1}}{f_{2}}=\frac{f_{1}}{f_{1}+\left(1-f_{1}\right) f_{1}}=\frac{1}{2-f_{1}}=\frac{1}{1+\varphi_{1}} ; \quad \mathrm{c}_{\mathrm{S}}=\frac{f_{2}-f_{1}}{f_{2}} \\
& =\frac{f_{2}-f_{1}}{f_{1}+\left(1-f_{1}\right) f_{1}}=\frac{\left(1-f_{1}\right) f_{1}}{f_{1}+\left(1-f_{1}\right) f_{1}}=\frac{1-f_{1}}{2-f_{1}}=\frac{\varphi_{1}}{1+\varphi_{1}} ;
\end{aligned}
$$

see Eq. (4). Furnas [18,19] called mixes of bimodal particles that obey these values of $c_{\mathrm{L}}$ and $c_{\mathrm{S}}$ "saturated mixtures," in such mixture the sufficient small particles are added to just fill the void fraction between the large particles. Indeed for $\varphi_{1}=0.50$ and $u \rightarrow \infty$, the lowest void fraction is obtained when the volume fractions of large and small particles tend to $2 / 3\left[=\left(1+\varphi_{1}\right)^{-1}\right]$ and $1 / 3\left[=\varphi_{1}\left(1+\varphi_{1}\right)^{-1}\right]$, respectively, see Eq. (5). In that case, $r$ tends to $2\left(=1 / \varphi_{1}\right)$ and the void fraction $h$ tends to $1 / 4$ [Table I], the latter corresponding to $\varphi_{1}^{2}$ [Eq. (4)].

One the other hand, for $u \rightarrow 1$, Fig. 1 and Table I indicate that that both $c_{\mathrm{L}}$ and $c_{\mathrm{S}}$ tend to $1 / 2$ (or $r$ to unity); i.e., for a maximum packing fraction, the volume fractions of both size groups become equal. In the past, in contrast to saturated mixes where $u$ tends to infinity, the packing behavior of bimodal mixes in the vicinity of a single-sized mix (i.e., when the two sizes tend to each other, that is, $u$ tends to unity) has hardly been examined. 
TABLE I. Mixing conditions for maximum bimodal packing fraction of spheres, derived from Ref. [18].

\begin{tabular}{cccccc}
\hline \hline$d_{\mathrm{L}} / d_{\mathrm{S}}(u)$ & $c_{\mathrm{L}}=k(u)$ & $c_{\mathrm{S}}=1-c_{\mathrm{L}}$ & $r=g(u)$ & $h[u, r=g(u)]$ & ${ }^{\prime} \log (\mathrm{g})$ \\
\hline 1 & 0.5 & 0.5 & 1 & 0.5 & - \\
2 & 0.52 & 0.48 & 1.083 & 0.474 & 0.115 \\
2.5 & 0.54 & 0.46 & 1.174 & 0.440 & 0.175 \\
3.33 & 0.64 & 0.36 & 1.778 & 0.412 & 0.478 \\
5 & 0.66 & 0.34 & 1.941 & 0.376 & 0.412 \\
10 & $\rightarrow 2 / 3$ & $\rightarrow 1 / 3$ & $\rightarrow 2$ & 0.328 & $\rightarrow 0.30$ \\
20 & $\rightarrow 2 / 3$ & $\rightarrow 1 / 3$ & $\rightarrow 2$ & 0.314 & $\rightarrow 0.23$ \\
50 & $\rightarrow 2 / 3$ & $\rightarrow 1 / 3$ & $\rightarrow 2$ & 0.270 & $\rightarrow 0.17$ \\
\hline \hline
\end{tabular}

Mangelsdorf and Washington [25] seem to be the only ones who experimentally examined the limit of $u=1$ more closely. They executed packing fraction experiments with a number of binary mixes of spheres, whereby the spheres had relatively small diameter ratios of 1.16 to 1.6. Even with the largest diameter ratio, there was no apparent asymmetry in contraction (void fraction reduction). Also from Fig. 1, one can conclude that even for $u=2$, only a slight asymmetry takes place. So, for $1 \leqslant u \leqslant 1.6$, Mangelsdorf and Washington [25] described the void fraction reduction with a symmetrical curve of the form $c_{\mathrm{L}}\left(1-c_{\mathrm{L}}\right)$. Their equation also implies that in the vicinity of equal sphere diameters ( $u$ tending to unity) maximum packing fraction is obtained for $c_{\mathrm{L}}=c_{\mathrm{S}}(=0.5)$, and hence $r=1$. The same trend can also be observed in Fig. 1. Monte Carlo simulations also indicate this symmetrical behavior for diameter ratios close to unity $[17,26]$. As will be explained in the following paragraph, also from a basic consideration of the gradients in bimodal void fraction at $u=1$ and $c_{\mathrm{L}}=c_{\mathrm{S}}=0.5(r=1)$, this conclusion of maximum descent in the direction of the unit vector $\left(u=1, c_{\mathrm{L}}=0\right)$ can be drawn.

In the vicinity of $u=1$, as depicted in Fig. 1, the bimodal void fraction is described with a Redlich and Kister type equation [27], which was derived to describe thermodynamically the excess energy involved with the mixing of liquids. From Fig. 1 it follows that along $\left(u=1,0 \leqslant c_{\mathrm{L}} \leqslant 1\right)$, or equivalently, along $(u=1,0 \leqslant r \leqslant \infty)$, the void fraction remains $\varphi_{1}$, physically this implies that particles are replaced by particles of identical size, i.e., maintaining a single-sized mixture [28]. As the gradient of the void fraction $h$ at $u=1$ and $c_{\mathrm{L}}=c_{\mathrm{S}}=0.5$ (or $r=1$ ) is zero in the direction of $c_{\mathrm{L}}$ (or $r$ ), the gradient will be largest perpendicular to this direction, i.e., in the direction of $u$. This feature of the gradient of the bimodal void fraction is also in line with the bimodal void fraction being symmetrical near $u=1$ and $c_{\mathrm{L}}=c_{\mathrm{S}}=0.5$ (or $r$ $=1)$.

In Table I, the values of $c_{\mathrm{L}}, c_{\mathrm{S}}$, and $r$ are given at which maximum packing fraction (void fraction $h$ is minimum) takes place versus the diameter ratio. These specific volume fractions $c_{\mathrm{L}}$ and $c_{\mathrm{S}}$ and their specific ratio $r$ depend on the size ratio $u$, and are therefore denoted as $r=g(u)$ and $c_{\mathrm{L}}$ $=k(u)$, with $g(u)$ and $k(\mathrm{u})$ being related by Eq. (1) as

$$
g(u)=\frac{k(u)}{1-k(u)}
$$

As discussed above, for $u \rightarrow 1, k(u)$ tends to $1 / 2$ and $g(u)$ to 1 , for $u \rightarrow \infty, k(u)$ tends of $2 / 3$ and $g(u)$ tends to 2 [Table I]. In Fig. $1, k(u)$ is included as well $(1 \leqslant u \leqslant 2.5)$, and in Fig. 2(a), $g(u)$ is set out versus $u(1 \leqslant u \leqslant 5)$. One can see that for RLP of spheres, beyond $u \approx 3-4$, the smaller spheres seem to fit in the interstices of the larger ones. For close fcc/hcp lattices this is the case for $u>2.4$ and for close bcc lattices for $u>6.5$.

\section{B. Discrete geometric packing}

Furnas [19] subsequently extended the discrete binary packing model to multimodal discrete packing. The major consideration is that the holes of the larger particles (characteristic size $d_{1}$ ) are filled with smaller particles $\left(d_{2}\right)$, whose voids in turn are filled with smaller ones $\left(d_{3}\right)$, and so on till the smallest diameter $d_{n}$, whereby the diameter ratio

$$
u=d_{1} / d_{2}=d_{2} / d_{3} \text { etc. }>u_{b} .
$$

As the interstices of the smaller particle are filled with smaller ones, the distribution of the particles is forming a geometrical progression. The number of fractions, $n$, readily follows from

$$
n=1+{ }^{u} \log \left(d_{1} / d_{n}\right) .
$$

In general, the packing fraction and void fraction of multiple mode distributions of $n$ size groups, with $n \geqslant 1$, then read

$$
f_{n}=1-\left(1-f_{1}\right)^{n} ; \quad \varphi_{n}=1-f_{n}=\left(1-f_{1}\right)^{n}=\varphi_{1}^{n} .
$$

The volume fraction of each size group $i(i=1,2, \ldots, n)$ in the mixture of $n$ size groups follows as:

$$
c_{i}=\frac{\varphi_{1}^{i-1}-\varphi_{1}^{i}}{f_{n}}=\frac{\varphi_{1}^{i-1}\left(1-\varphi_{1}\right)}{1-\varphi_{1}^{n}} .
$$

It can easily be verified that $c_{1}+c_{2}+\cdots+c_{n-1}+c_{n}=1$. Equation (10) indicates that the amount of adjacent size groups has a constant ratio, 


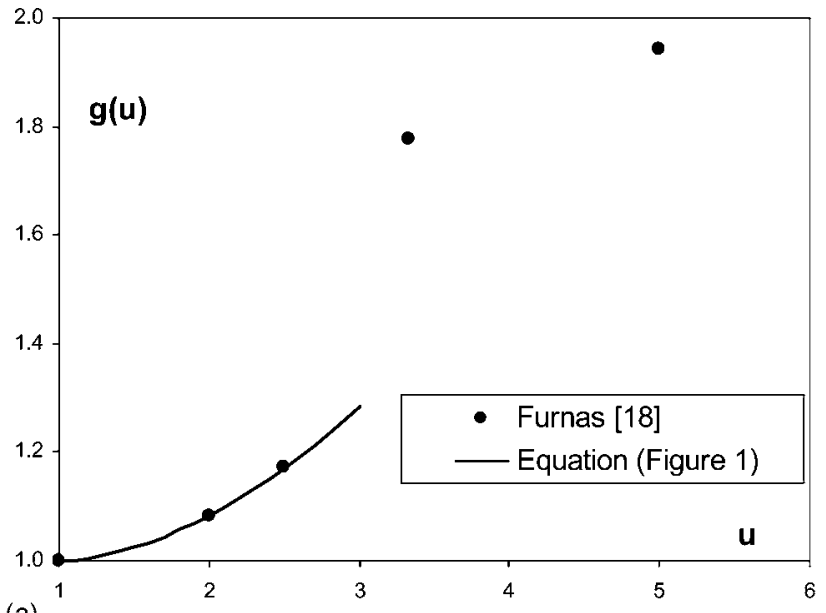

(a)

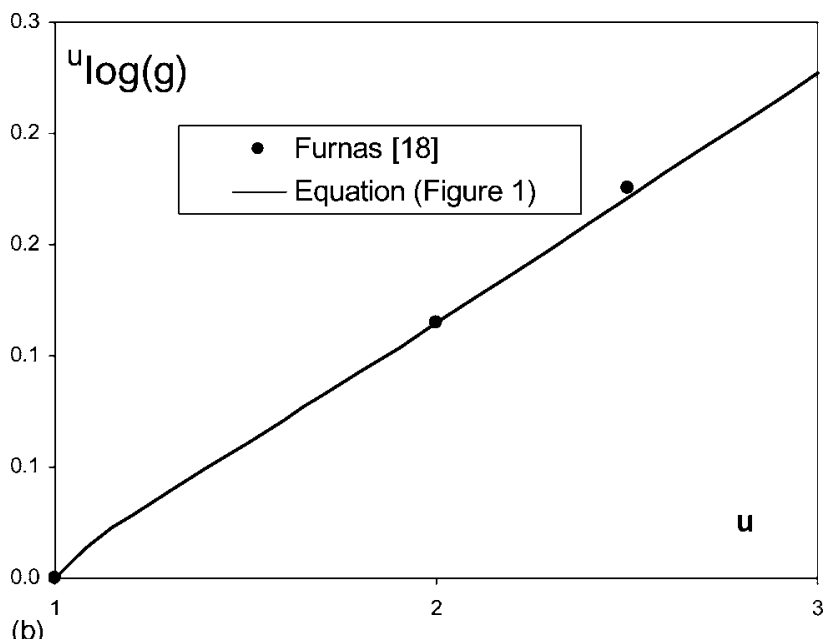

(b)

FIG. 2. (a) Concentration ratio $r$ as a function of the size ratio $u$ at maximum packing fraction $[r=g(u)]$, using data of Table I, and computed with $g(u)=k(u) /[1-k(u)]$ for $1 \leqslant u \leqslant 3$ using the formula of $k(u)$ given in Fig. 1. (b) Distribution modulus $\alpha$ as a function of the size ratio $u$ at maximum packing fraction $\left[\alpha={ }^{u} \log (g)\right]$, computed with $g(u)=k(u) /[1-k(u)]$ for $1 \leqslant u \leqslant 3$ using the formula of $k(u)$ given in Fig. 1. [These computed values of $k, g$, and $\alpha$ are listed in Table II.]

$$
r=\frac{c_{i}}{c_{i+1}}=\frac{1}{\varphi_{1}},
$$

as is also the case for the particle size ratio of each subsequent size group $\left(u_{b}\right)$, i.e., a geometric progression is obtained. For the special case of a bimodal mixture $(n=2)$, Eqs. (9) and (10) obviously transform into Eqs. (4) and (5), respectively, when $i=1$ and 2 are substituted.

\section{Continuous geometric packing}

For packing of a continuous particle-size distribution (PSD), Andreasen and Andersen [20] originally proposed the semiempirical formula for the cumulative finer fraction (or cumulative distribution function)
TABLE II. Mixing conditions for maximum bimodal packing fraction of spheres, computed using the formulas given in Fig. 1. The value of ${ }^{u} \log (g)$ for $u=r=1$ is obtained by taking the limit.

\begin{tabular}{cccccc}
\hline \hline$d_{\mathrm{L}} / d_{\mathrm{S}}(u)$ & $c_{\mathrm{L}}=k(u)$ & $c_{\mathrm{S}}=1-c_{\mathrm{L}}$ & $r=g(u)$ & $h[u, r=g(u)]$ & ${ }^{u} \log (g)$ \\
\hline 1 & 0.5 & 0.5 & 1 & 0.5 & 0 \\
$\sqrt{2}$ & 0.504 & 0.496 & 1.018 & 0.487 & 0.052 \\
2 & 0.520 & 0.480 & 1.083 & 0.469 & 0.115 \\
2.5 & 0.539 & 0.461 & 1.170 & 0.453 & 0.171 \\
3.33 & 0.578 & 0.422 & 1.370 & 0.425 & 0.262 \\
\hline \hline & $F(d)=\left[\frac{d}{d_{\max }}\right] \alpha$
\end{tabular}

by formulating and solving the equation

$$
\frac{d F}{d(\ln d)}=\alpha F,
$$

and invoking boundary condition

$$
F\left(d_{\text {max }}\right)=1 .
$$

Equation (13) is based on the insight that a maximum packing fraction is achieved when coarser fractions are placed in such quantities that they represent in each size class the same fraction $\alpha$ of the quantity which was present before. The particles sizes are such that the sizes $d$ of successive classes form a geometrical progression, so that the particle size increases with $d(\log d)$. This formulation, however, does not permit a minimum particle size, which will always be the case (e.g., see Refs. [22,29]).

\section{RELATING DISCRETE AND CONTINUOUS GEOMETRIC PACKINGS}

In this section, the discrete geometric particle packing and continuous geometric particle packing are mathematically coupled. It will be seen that the bimodal discrete packing, in the limit of the size ratio $u$ tending to unity, plays a key role in this analysis.

\section{A. Interacting discrete geometric packing}

The geometrical considerations learn that for noninteracting discrete particles (i.e., $u>u_{b}$ ) size ratios $u$ are constant, and that the concentrations of subsequent sizes have a constant ratio $\left(1 / \varphi_{1}\right)$; see Eq. (11). As explained in the previous section, nondisturbance prevails when $u\left(=d_{i} / d_{i+1}\right)$ exceeds $u_{b}(\approx 7-10)$. The cumulative finer function $F$ of such discrete packing consists of multiple Heaviside functions. At each $d_{i}, F$ increases with $c_{i}$, whereby $c_{i}$ follows from Eq. (10). In Fig. 3(a) this is explained graphically for a bimodal packing. In a frequency distribution graph, at each size group $d_{i}$, the population is given by $c_{i} \delta\left(d_{i}\right), \delta(x)$ being the Dirac function. As $d_{i} / d_{i+1}=u_{b}$ and $c_{i} / c_{i+1}=r=1 / \varphi_{1}$ [Eq. (11)], for multicomponent mixes it is convenient to set out $c_{i}$ and $d_{i}$ in a double logarithmic graph, as both ${ }^{a} \log d_{i}-{ }^{a} \log d_{i+1}$ and ${ }^{b} \log c_{i}-{ }^{b} \log c_{i+1}$ are constant, being ${ }^{a} \log u_{b}$ and ${ }^{b} \log \phi_{1}^{-1}$, re- 


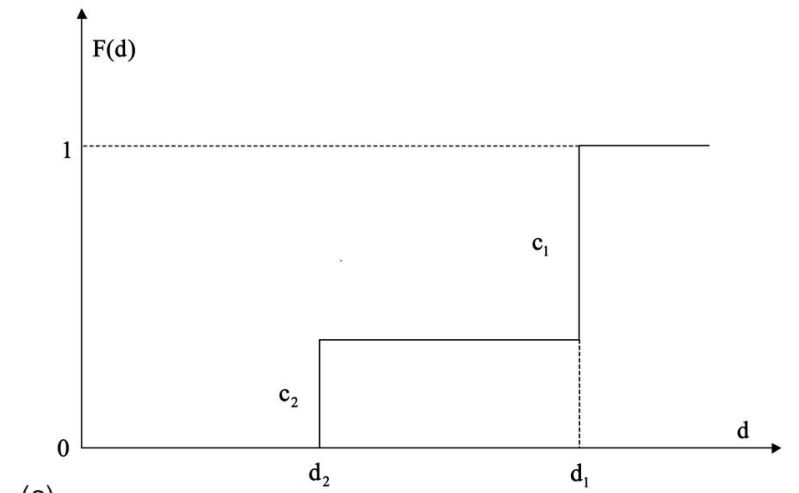

(a)
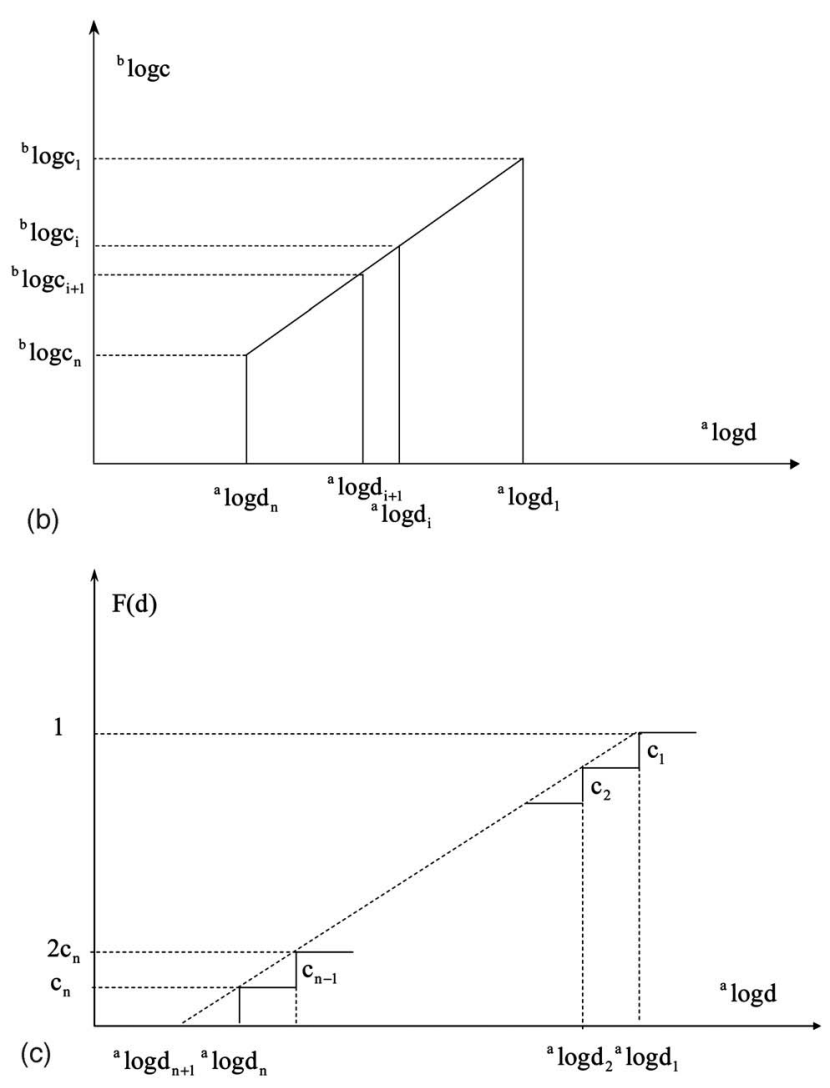

FIG. 3. (a) Cumulative finer fraction $F$ for a bimodal mix $\left(c_{2}\right.$, $d_{2}, c_{1}$, and $d_{2}$ correspond to $c_{\mathrm{S}}, d_{\mathrm{S}}, c_{\mathrm{L}}$, and $d_{\mathrm{L}}$, respectively). (b) The logarithm of concentrations versus the logarithm of the particle size for a geometric discrete distribution. (c) Cumulative finer fraction $F$ versus the logarithm of the particle size for a discrete geometric discrete distribution (step function) and for a geometric distribution composed with sieve fractions that have continuous populations (diagonal dotted line).

spectively. Figure 3(b) reflects such a distribution consisting of $n$ sizes between $d_{n}$ and $d_{1}$, for which both the size ratio and the quantity ratio of each size group are constant.

One can also construct a polydisperse geometric distribution whereby $u<u_{b}$, so that the particles will interact and the size ratio and quantity ratio of each size group is no longer prescribed by $u_{b}$ and $1 / \varphi_{1}$, respectively. In that case, the $r$ that pertains to a maximum packing fraction, $g$, depends on $u$ [e.g., see Fig. 2(a)], and tends to unity when $u$ tends to unity, viz. the sizes and the volume fractions of small and large particles become equal (see previous section).

Now, the size of group $i$ is related to the minimum and maximum particle size by

$$
d_{i}=d_{n}\left(u^{n-i}\right)=d_{1}\left(u^{1-i}\right),
$$

as

$$
\frac{d_{i}}{d_{i+1}}=u
$$

Taking the logarithm of the particle size, a linear relation is obtained

$$
\begin{aligned}
{ }^{a} \log d_{i} & ={ }^{a} \log d_{n}+\left(\frac{n-i}{n-1}\right)\left({ }^{a} \log d_{1}-{ }^{a} \log d_{n}\right) \\
& ={ }^{a} \log d_{n}+(n-i){ }^{a} \log u,
\end{aligned}
$$

as

$$
{ }^{a} \log u=\left(\frac{1}{n-1}\right)\left({ }^{a} \log d_{1}-{ }^{a} \log d_{n}\right),
$$

see Eq. (15).

Furthermore, also the concentration (or quantity) ratio of subsequent size groups is constant

$$
\frac{c_{i}}{c_{i+1}}=r
$$

or

$$
\mathrm{c}_{\mathrm{i}}=c_{n}\left(r^{n-i}\right)=c_{1}\left(r^{1-i}\right) .
$$

Again, taking the logarithm of the concentration ratio, a linear relation is obtained

$$
\begin{aligned}
{ }^{b} \log c_{i} & ={ }^{b} \log c_{n}+\left(\frac{n-i}{n-1}\right)\left({ }^{b} \log c_{1}-{ }^{b} \log c_{n}\right) \\
& ={ }^{b} \log c_{n}+(n-i){ }^{b} \log r,
\end{aligned}
$$

as

$$
{ }^{b} \log r=\left(\frac{1}{n-1}\right)\left({ }^{b} \log c_{1}-{ }^{b} \log c_{n}\right),
$$

see Eq. (20). For both arbitrary logarithm bases hold $a>0$ and $b>0$. Again Fig. 3(b) can be used to illustrate that, in view of Eqs. (17) and (21), in the double logarithmic graph the distance between subsequent particle sizes is constant, as well as the differences between subsequent concentrations.

The cumulative finer fraction at $d=d_{i}$ follows as

$$
F\left(d_{i}\right)=\frac{\sum_{i}^{n} c_{i}}{\sum_{1}^{n} c_{i}}=\frac{c_{i}+c_{i+1}+\ldots+c_{n-1}+c_{n}}{c_{1}+c_{2}+\ldots+c_{n-1}+c_{n}} .
$$




$$
\begin{aligned}
F\left(d_{i}\right) & =\frac{r^{n-i} c_{n}+r^{n-i-1} c_{n}+\ldots+r c_{n}+c_{n}}{r^{n-1} c_{n}+r^{n-2} c_{n}+\ldots+r c_{n}+c_{n}} \\
& =\frac{1+r+r^{2}+\ldots+r^{n-i}}{1+r+r^{2}+\ldots+r^{n-1}}=\frac{r^{n-i+1}-1}{r^{n}-1} .
\end{aligned}
$$

Note that in the saturated bimodal system $(n=2)$ [see Fig. 3(a)], $F\left(d_{2}\right)\left(=c_{2}=c_{S}\right)$ amounts $1 /(1+r)$, whereby $r=1 / \varphi_{1}$ [Eq. (11)]. Obviously, $F\left(d_{1}\right)=1$, and $F\left(d_{1}\right)-F\left(d_{2}\right)$ corresponds to $c_{1}\left(=c_{L}\right)$. This expression also features that at $i=n$, i.e., $d_{i}=d_{n}, F>0$. This is a consequence of the fact that the first particles are added at this smallest particle size. Furthermore, Eq. (24) reveals that that $F=0$ at $i=n+1$, i.e., at $d$ $=d_{n+1}$ whereby this size also obeys Eq. (16), i.e., $d_{n+1}$ $=d_{n} / u$.

Accordingly, the size ratio is defined as

$$
y=\frac{d_{1}}{d_{n+1}}=u^{n} ; \quad d_{i}=d_{1}(y)^{(1-i) / n} ; \quad d_{i}=d_{n+1}(y)^{(n-i+1) / n},
$$

so that the number of size groups follows as

$$
n={ }^{u} \log y={ }^{u} \log \left[\frac{d_{1}}{d_{n+1}}\right],
$$

which is compatible with Eq. (8) as $d_{n+1}=d_{n} / u$ [Eq. (16)]. In order to decouple a particular size $d_{i}$ from the number of size groups, $n-i+1$ is related to $d_{i}$ by using Eqs. (25) and (26),

$$
\begin{aligned}
n-i+1 & =n\left(\frac{{ }^{a} \log \left(d_{i} / d_{n+1}\right)}{{ }^{a} \log \left(d_{1} / d_{n+1}\right)}\right) \\
& ={ }^{u} \log \left[\frac{d_{1}}{d_{n+1}}\right]\left(d_{i} / d_{n+1}\right) \log ^{-1}\left[\frac{d_{1}}{d_{n+1}}\right]={ }^{u} \log \left[\frac{d_{i}}{d_{n+1}}\right] .
\end{aligned}
$$

Substitution of Eqs. (26) and (27) into Eq. (24) yields

$$
F\left(d_{i}\right)=\frac{r^{u^{u} \log \left(d_{i} / d_{n+1}\right)}-1}{r^{u \log \left(d_{1} / d_{n+1}\right)}-1},
$$

which indeed covers $F=0$ (at $\left.d_{i}=d_{n+1}\right)$ to $F=1 \quad\left(\right.$ at $\left.d_{i}=d_{1}\right)$. This equation is rewritten by using mathematics

$$
r^{u} \log \left(d_{i} / d_{n+1}\right)=\left[\frac{d_{i}}{d_{n+1}}\right]^{u \log r} ; r^{u \log \left(d_{1} / d_{n+1}\right)}=\left[\frac{d_{1}}{d_{n+1}}\right]^{u} \log r
$$

yielding as discrete cumulative finer fraction at discrete sizes $d_{i}=d_{n+1}, d_{n}, \ldots, d_{2}, d_{1}$,

$$
F\left(d_{i}\right)=\frac{d_{i}^{\alpha}-d_{n+1}^{\alpha}}{d_{1}^{\alpha}-d_{n+1}^{\alpha}}
$$

with

$$
\alpha={ }^{u} \log r .
$$

So, for when polydisperse discrete particle are geometrically packed, the cumulative distribution follows Eq. (30). For a given size ratio $u$, e.g., $u=2$, it follows that a maximum packing fraction can be obtained by considering the bimodal packing, taking the pertaining concentration ratio $r=g(u)$ and computing $\alpha$ according to Eq. (31). In Tables I and II, set out in Figs. 2(a) and 2(b), one can find these specific $r=g(u)$ and $\alpha$, respectively, as a function of $u$ for RLP of spheres. Due to the nature of the bimodal packing, for $u>1$ a maximum packing fraction occurs when $c_{\mathrm{L}}>c_{\mathrm{S}}$ (and hence $r>1$ ), and also $\alpha>1$. In Fig. 2(b), the exponent pertaining to maximum discrete packing for RLP of spheres is given, based on Eq. (31) and the expression for $g(u)$ given in Fig. 1. One can see an almost linear increase in $\alpha$ for increasing size ratio $u$, and in the limit of $u=1$ (i.e., continuous distribution), $\alpha$ tends to zero. This result is based on the Redlich and Kister expression given in Fig. 1. In what follows, the value of $\alpha$ in the general limit of $u \rightarrow 1$ and $r \rightarrow$ is determined, i.e., a continuous geometric distribution is obtained, and it is demonstrated that then indeed $\alpha=0$ corresponds to maximum packing.

\section{B. Transformation into continuous geometric packing}

For a given size ratio $y$, in the limit of $n \rightarrow \infty$, it follows that $u$ (or $d_{i} / d_{i+1}$ ) tends to unity, for Eq. (25) yields

$$
u=y^{1 / n}=1+\frac{1}{n} \ln y+\mathrm{O}\left(\frac{1}{n^{2}}\right) .
$$

In such continous case also the size ratio $r$ tends to unity. This is illustrated by Figs. 1 and 2(a), in which $c_{\mathrm{L}}=k(u)$ and $r=g(u)$ (that is, $c_{\mathrm{L}}$ and $r$ belong to the maximum packing fraction) are set out against $u$ [the values taken from Tables I and II], respectively. Figure 2(a) is based on bimodal data $\left[g(u)=c_{\mathrm{L}} / c_{\mathrm{S}}\right.$ and $\left.u=d_{\mathrm{L}} / d_{\mathrm{S}}\right]$, for the multiple discrete packing considered here $u$ corresponds to $d_{i} / d_{i+1}$, and $r$ corresponds to $c_{i} / c_{i+1}$.

Application of Eq. (32) to Eq. (31) yields the limit

$$
\lim _{u \rightarrow 1} \alpha=\lim _{u \rightarrow 1}{ }^{u} \log r=\lim _{u \rightarrow 1}\left(\frac{{ }^{a} \log u}{{ }^{a} \log r}\right)=\left.\frac{d r}{d u}\right|_{u=1},
$$

with logarithm base $a>0$. In such case, a continuous distribution is obtained,

$$
\begin{gathered}
F(d)=\frac{d^{\alpha}-d_{\text {min }}^{\alpha}}{d_{\text {max }}^{\alpha}-d_{\text {min }}^{\alpha}} \quad \alpha \neq 0, \\
F(d)=\frac{\ln d-\ln d_{\text {min }}}{\ln d_{\text {max }}-\ln d_{\text {min }}} \quad \alpha=0 .
\end{gathered}
$$

The (now) continuous $d$ replaces the discrete $d_{i}, d_{\max }$ the $d_{1}$, and $d_{\min }$ the $d_{n+1}$, respectively. Note that the four logarithms in Eq. (35) can have any base $a>0$; here, the natural logarithm is selected arbitrarily. Equation (35) follows directly from taking the limit $\alpha \rightarrow 0$ of Eqs. (30) and/or (34). It also follows from a similar derivation as executed above, but now with invoking that all concentrations are identical. Note that for this distribution the population consists of $n$ Dirac functions, $c_{n} \delta\left(d_{n}\right), c_{n-1} \delta\left(d_{n-1}\right), \cdots, c_{2} \delta\left(d_{2}\right), c_{1} \delta\left(d_{1}\right)$. This more 
basic case is addressed below to illustrate the reasoning followed previously, and that resulted in Eq. (34).

In this case $c_{n}=c_{n-1}=\cdots=c_{2}=c_{1}$, in a single logarithmic graph, the cumulative finer function now is a multiple Heaviside function with equal increments [Fig. 3(c)]. Hence, Eq. (24) yields

$$
F\left(d_{i}\right)=\frac{n-i+1}{n} .
$$

Again, it follows that $F=0$ for $i=n+1$, or $d=d_{n+1}=d_{n} / u$. In Fig. 3(c), this particle size is added. So by letting $i$ range from $n+1$ up to 1 , the cumulative finer function $F$ of the discrete packing ranges from zero to unity. Also now $n-i+1$ is expressed in $d_{i}$ (and $n$ eliminated) by substitution of Eq. (26) into Eq. (36), yielding

$$
F\left(d_{i}\right)=\frac{{ }^{a} \log d_{i}-{ }^{a} \log d_{n+1}}{{ }^{a} \log d_{1}-{ }^{a} \log d_{n+1}} .
$$

In the limit of $n \rightarrow \infty$, indeed this discrete distribution transforms into continuous distribution (35). Hence, an infinite number of identical discrete increments (or integration/ summation of multiple Dirac population functions) is turned into a continuous function, as has been performed above for the more complex case of $\alpha \neq 0$, for which Eq. (34) holds. Subsequently, the population (or frequency distribution) of the continuous power law distribution is obtained by differentiating Eqs. (34) and (35) with respect to $d$,

$$
\begin{gathered}
p(d)=\frac{d F}{d d}=\frac{\alpha d^{\alpha-1}}{d_{\text {max }}^{\alpha}-d_{\text {min }}^{\alpha}} \quad \alpha \neq 0, \\
p(d)=\frac{d F}{d d}=\frac{d^{-1}}{\ln d_{\max }-\ln d_{\min }} \quad \alpha=0 .
\end{gathered}
$$

\section{Relation with composed distributions}

Both derivations ( $\alpha=0$ and $\alpha \neq 0)$ lead to discrete distribution functions [Eqs. (30) and (37)] that start at $d=d_{n+1}$. The underlying populations (multiple delta functions) can also be generalized to multiple continuous populations, whereby the concentrations $c_{i}$ hold for all particles sized between $d_{i+1}$ and $d_{i}$. These particle classes are, for instance, obtained when a particulate material is sieved, whereby particles between $d_{i+1}$ and $d_{i}$ are sieve class $i, u$ is then the sieve size ratio, and the density function $p_{i}$ of each sieve fraction is not explicitly known [30]. So, $c_{1}$ represents the amount of particles with sizes lying between $d_{2}$ and $d_{1}, c_{n}$ represents the particles ranging from $d_{n+1}$ to $d_{n}$, etc. The discrete distribution shown in Fig. 3(c) actually reflects an extreme case for which all particles of a sieve class possess the maximum size of the class $\left[p_{i}=c_{i} \delta\left(d_{i}\right)\right]$. The same packing fraction is obtained when all particles from a sieve class would have the minimum size $\left(d_{i+1}=d_{i} u^{-1}\right)$ instead of the maximum $\left(d_{i}\right)$. In other words, all particles are reduced in size by a factor $u^{-1}$; in Figs. 3(b) and 3(c), this corresponds when the graph is shifted with ${ }^{a} \log u$ to the left (so $c_{1}$ pertains to $d_{2}, c_{n}$ to $d_{n+1}$, etc). The dotted straight line in Fig. 3(c), on the other hand, corresponds to continuous populations of each sieve class. In other words, when the population of each class is continuous (and not discrete), the cumulative finer function becomes a continuous function (instead of a multiple Heaviside function). Furthermore, when the concentration ratio of the sieve classes, $r$, is identical to the one of the discrete distribution, the cumulative finer function has the same value at all discrete values $d=d_{i}$. So, in that case $F\left(d_{i}\right)$ is identical [compare multiple step function and dotted line of Fig. 3(c)] and is governed again by Eqs. (30) and (37). In Ref. [29] the special case of sieve populations being a power law function is addressed as well, using Eqs. (38) and (39).

The analogy between multiple discrete and multiple continuous populations inspired several researchers to create quasi power law distributions whereby the sieve amount ratio, $r$ (like the discrete particle concentration ratio), is constant. Furnas [19] and Anderegg [31] found for sieves having a ratio $u$ of $\sqrt{2}, r=1.10$ gave minimum voids for densely packed (irregular) aggregates, and for sieves with $\mathrm{u}=2$, $r=1.20$ gave a densest packing fraction. Also in Ref. [23] a constant $r\left(10^{0.06} \approx 1.15\right)$ is recommended for dense cement and aggregate packing using a sieve set with constant size ratio $u\left(10^{0.3} \approx 2\right.$, "série de Renard"). Substituting the abovementioned $(u=\sqrt{2}, \quad r=1.10), \quad(u=2, \quad r=1.20)$, and $(u=2$, $r=1.15$ ) in Eq. (31) yields $\alpha=0.28,0.26$, and 0.20 , respectively. All these exponents of the distribution curve, which hold for densely packed angular particles with unknown population, are positive and in the same range as discrete loosely packed spheres (to which the bimodal data can be applied).

For these loose packings of spheres, $u$ and $r=g(u)$ are included in Tables I and II, revealing that $(u=\sqrt{2}$, $r=1.018)$ and ( $u=2, r=1.083)$ yielding $\alpha=0.052$ and 0.115 , result in a maximum packing fraction. This bimodal information is applicable to multiple discrete packings, but cannot simply be applied to multiple packings of adjacent (continuous) sieve classes with a given (unknown) population, though their $\alpha$ values are positive and their magnitude do not differ that much. Sieve classes that have a large size difference, on the other hand, behave identically as discrete packings with large size ratios $\left(u>u_{b}\right)$. Sohn and Moreland [16] measured that binary mixtures of continuous (normal and log normal) distributions tend to saturated state when the ratio of characteristic particle size tends to infinity. In that case the packing/void fraction is governed again by Eq. (4), whereby $f_{1} / \varphi_{1}$ stands for the values of each single continuous distribution.

\section{Relation with previous work}

Equation (34) was also proposed in Ref. [22], who modified the equation derived by Andreasen and Andersen [20] that was discussed in the previous section, by introducing a minimum particle size in the distribution. For many years, Eq. (34) is also in use in mining industry for describing the PSD of crushed rocks [32]. Actually, following the geometric reasoning of Andreasen and Andersen [20] (see previous section), this would result in the following equation for the population: 

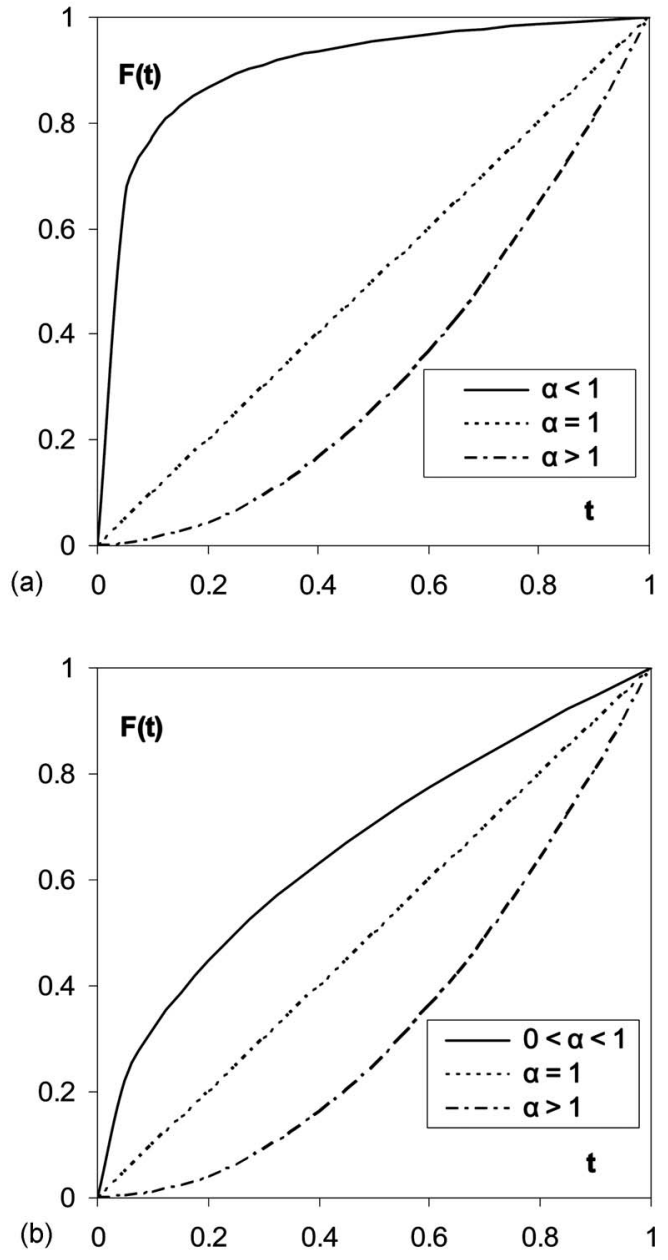

FIG. 4. (a) Cumulative finer fraction $F$ versus dimension particle size $t$ according to Eq. (12) with (as examples) $d_{\min }=0$ $(1 / y=0)$ for $\alpha=0.5, \alpha=1$, and $\alpha=2$. (b) Cumulative finer fraction $F$ versus dimension particle size $t$ according to Eq. (35) with (as example) $y=100$ for $\alpha=-0.5, \alpha=1$, and $\alpha=2$.

$$
\frac{d p}{d(\ln d)}=(\alpha-1) p
$$

instead of Eq. (13). Integrating this equation twice with respect to $d$ to obtain $F(d)$, applying boundary conditions Eq. (14) and

$$
F\left(d_{\min }\right)=0,
$$

then yield Eqs. (34) and (35) indeed. So, Eqs. (13) and (40) both yield power law distributions for $F$ and $p$, but the formulation and solving of the latter enables the existence of a smallest particle size in the mix. Furthermore, Eqs. (34) and (35), in contrast to Eq. (12), also permit negative values of the distribution exponent $\alpha$ in the PSD. Figures 4(a) and 4(b) explain the difference in nature of Eq. (12) and Eqs. (34) and (35), respectively, with regard to the value of $\alpha$. In these figures, $F$ is set out versus the dimensionless particle size $t$, defined as

$$
t=\frac{d-d_{\min }}{d_{\max }-d_{\min }} .
$$

The modified "Andreasen and Andersen" PSDs [Figure 4(a)] are convex for $\alpha<1$ and concave for $\alpha>1$. The same holds for the original Andreasen and Andersen PSD [Figure 4(b)], which features the limitation $\alpha>0$. Note that for $\alpha \rightarrow \infty$ and for $\alpha \rightarrow-\infty$, Eq. (34) tends to a monosized distribution with particle size $d_{\min }$ and $d_{\max }$, respectively.

In an earlier attempt to relate discrete and continuous geometric packings, Zheng et al. [21] derived Eq. (34) with an exponent,

$$
\alpha={ }^{10} \log \varphi_{1}^{-1}
$$

which is based (and valid only) on saturated discrete packings. The value $u=10$ was selected as size ratio for which undisturbed packing can be assumed (so $u_{b}=10$ ), for which $r=1 / \varphi_{1}$ indeed (Sec. II). Funk and Dinger [22] postulated Eqs. (31) and (34), based on graphical considerations, and applied it to continuous packings. In all these elaborations the limit as pointed out in Eq. (32) was, however, not considered. This limit is required to transform the polydisperse discrete packing mathematically into a continuous packing, and to unambiguously relate its distribution modulus to the bimodal packing characteristics [especially its $r(u)$ ], discussed in more detail below.

Equation (33) reveals that the exponent $\alpha$ in the distributions [see Eqs. (34), (35), (38), and (39)] corresponds to the gradient of the ratio $c_{\mathrm{L}} / c_{\mathrm{S}}(r)$ in a bimodal system for $d_{\mathrm{L}} / d_{\mathrm{S}}$ (u) tending to unity. To describe $r$ in the vicinity of $u=1$, the Taylor expansion of $r$ at $u=1$ is given as

$$
\begin{aligned}
r(u) & =r(1)+\alpha(u-1)+\mathrm{O}\left((u-1)^{2}\right) \\
& =1+\alpha(u-1)+\mathrm{O}\left((u-1)^{2}\right),
\end{aligned}
$$

It was concluded in the previous section that the steepest reduction in void fraction, i.e., highest packing fraction, is encountered in the direction of $u$, perpendicular to the direction of $r$ (or $c_{\mathrm{L}}$ ) [see Figs. 1 and 2(a)], designated as $r=g(u)$ and $c_{\mathrm{L}}=k(u)$. From Eq. (33) this implies that in such case $\alpha=0$. So, combining the information on bimodal packings in the limit of equal sizes and the present continuum approach, it follows that a power law packing with $\alpha=0$ results in the densest packing fraction [i.e., Eq. (35)]. In Sec. IV the void fraction of power law packings, which depends on particle shape, mode of packing (e.g., loose, close), size width $y$, and distribution modulus $\alpha$, is quantified explicitly.

\section{VOID FRACTION OF GEOMETRIC PACKINGS}

In Sec. II the void fraction of multiple saturated discrete particle packings was given [Eq. (9)]. Here, the void fraction of the polydisperse continuous (power law) packing is addressed. In Sec. III it was demonstrated that in the limit of infinitesimal increments the multimodal discrete packing transforms to the power law packing, whereby the distribution modulus $\alpha$ follows from large/small component concentration in the discrete bimodal packing. It furthermore followed that a maximum packing fraction is obtained for 
TABLE III. Mixing conditions for maximum bimodal packing fraction of spheres extracted from Fig. 5 of Ref. [24].

\begin{tabular}{cc}
\hline \hline$d_{\mathrm{L}} / d_{\mathrm{S}}(u)$ & $h[u, r=g(u)]$ \\
\hline 1 & 0.375 \\
3.44 & 0.296 \\
4.77 & 0.256 \\
5.54 & 0.227 \\
6.53 & 0.203 \\
6.62 & 0.217 \\
6.88 & 0.225 \\
9.38 & 0.189 \\
9.54 & 0.178 \\
11.3 & 0.174 \\
16.5 & 0.169 \\
19.1 & 0.165 \\
77.5 & 0.155 \\
\hline \hline
\end{tabular}

$\alpha=0$, but the magnitude of the void fraction as such was not specified. Here it is shown that the infinite particle sizes approach as followed in the previous section can be employed to derive the void fraction of the power law distribution.

\section{A. Interacting discrete geometric packing}

Figure 3(b) reflects the geometric distribution of nondisturbing discrete particles when one assumes that $u>u_{b}$. The number of sizes between $d_{n}$ and $d_{1}$ follows from Eq. (8). The void fraction is obtained by combining Eqs. (8) and (9),

$$
\varphi=\varphi_{1}^{n}=\varphi_{1} \cdot \varphi_{1}^{u_{1} \log \left(d_{1} / d_{n}\right)}=\varphi_{1}\left(\frac{d_{1}}{d_{n}}\right)^{u \log \varphi_{1}}
$$

From this equation, one can see that the void fraction is reduced proportionally to the number of size groups minus one, and is in one part of the packing the same as in any other part. When the size ratio $u$ between the adjacent sizes is smaller than $u_{b}$, this perfect packing of smaller particles in the voids of the larger ones does not hold anymore, but also in this case the void fraction reduction involved with the size ratio of adjacent size groups (of constant ratio) is the same in any part of the packing. This is also confirmed by experimental results $[19,23,31]$.

As a first step, the void reduction involved with bimodal packing is analyzed in more detail, in particular its void fraction as function of concentration ratio and size ratio. In Sec. II it was explained that the void fraction $h(u, r)$ of such packings range from $\varphi_{1}^{2}$ (saturated) to $\varphi_{1}$ (monosized). From Table I, one can see that for the bimodal system of loosely packed spheres the void fraction becomes larger than 0.25 $\left(=\varphi_{1}^{2}\right)$ when $u<u_{b}$ (packing fraction becomes less), and tends to $0.5\left(=\varphi_{1}\right)$ when $u$ tends to unity. In Table III other data [24] is included, pertaining to densely packed spheres. In this vibrated system the unimodal void fraction of spheres is $0.375\left(\varphi_{1}\right)$, which is close to the minimum achievable (0.36, see Secs. I and II). For large size ratios it tends to

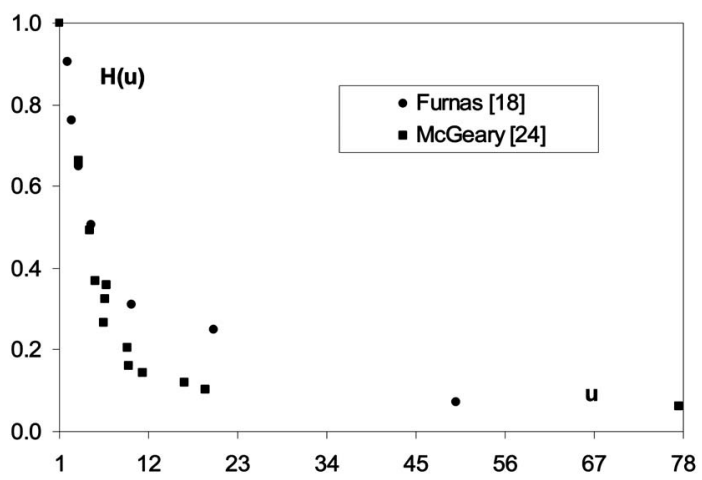

FIG. 5. Scaled void fraction of bimodal system at composition of maximum packing fraction $[H(u, r=g(u)]$ using data of Tables I and II.

$0.141\left(=\varphi_{1}^{2}\right)$, and for smaller $u$, the void fraction tends to its monosized value. To plot the data pertaining to the two different $\varphi_{1}$ in one graph, in Fig. 5 the scaled void fraction at maximum packing,

$$
H(u)=\frac{h[u, r=g(u)]-\varphi_{1}^{2}}{\varphi_{1}\left(1-\varphi_{1}\right)}
$$

is set out. Though the single-sized void fractions are different because of the two different modes of packing, it can be seen that $H(u)$ of both modes run down very similarly.

Considering the bimodal packing it follows that the monosized void fraction $\varphi_{1}$ is reduced with a factor $h / \varphi_{1}$ when a second smaller fraction is added, whereby $h(u, r)$ can range between $\varphi_{1}$ and $\varphi_{1}^{2}$ [or $H(u)$ from unity to zero]. For a bimodal system $h(u, r)$ holds, for a system with $n$ size groups, analogous to Eq. (45), holds

$$
\varphi=\varphi_{1}\left[\frac{h(u, r)}{\varphi_{1}}\right]^{n-1}
$$

A maximum packing fraction is obtained when $h$ is minimal, so when for a given $u, r$ is governed by $g$ (Figs. 1 and 2 for RLP of spheres).

\section{B. Transformation into continuous geometric packing}

Now the effect of adding an infinite number of size groups, to obtain a continuous packing, on void fraction can be quantified. Adding more size groups to the mix will reduce the void fraction. But on the other hand, its effect is less as for a given size width $y$ the size ratio of adjacent groups (i.e., $u$ ) tends to unity and the resulting void fraction of adjacent size groups, governed by $h(u, r)$, tends to $\varphi_{1}$. In the foregoing it was seen that in the limit of $n \rightarrow \infty$, it follows that both $u$ and $r$ tend to unity. Using the Taylor expansion of $h$ in the vicinity of $(u=1, r=1)$ in the direction of the unit vector $(u=\cos \gamma, r=\sin \gamma)$, see Fig. 6, and applying Eqs. (32) and (44) yields 


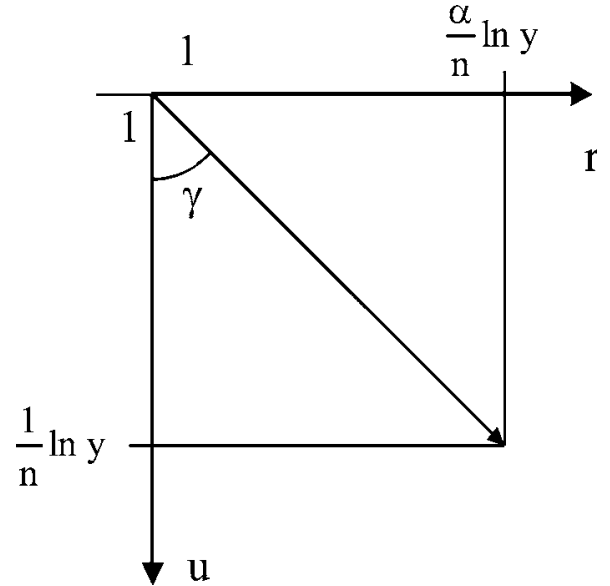

FIG. 6. Relation between gradients in $u$ and $r$ at $(u=1, r=1)$ and definition of $\gamma$.

$$
\begin{aligned}
h(u, r)= & h\left[1+\frac{1}{n} \ln y+\mathrm{O}\left(1 / n^{2}\right), 1+\frac{\alpha}{n} \ln y+\mathrm{O}\left(1 / \mathrm{n}^{2}\right)\right] \\
& \rightarrow h(1,1)+\left.\frac{\cos \gamma \ln y}{n} \frac{d h}{d u}\right|_{u=1, r=1} \\
& +\left.\frac{\sin \gamma \alpha \ln y}{n} \frac{d h}{d r}\right|_{u=1, r=1}+\mathrm{O}\left(\frac{1}{n^{2}}\right) \\
= & \varphi_{1}-\frac{\beta \varphi_{1}\left(1-\varphi_{1}\right) \cos \gamma \ln y}{n}+\mathrm{O}\left(\frac{1}{n^{2}}\right)
\end{aligned}
$$

in which the following directional derivatives are introduced at $(u=1, r=1)$ :

$$
\begin{gathered}
\beta=-\left.\frac{d H}{d u}\right|_{u=1, r=1}=-\left.\frac{1}{\varphi_{1}\left(1-\varphi_{1}\right)} \frac{d h}{d u}\right|_{u=1, r=1} ; \\
\left.\frac{d H}{d r}\right|_{u=1, r=1}=\left.\frac{d h}{d r}\right|_{u=1, r=1}=0 .
\end{gathered}
$$

From Fig. 6 it follows that

$$
\cos \gamma=\frac{1}{1+\alpha^{2}} .
$$

It should be realized that in Eq. (49), $\beta$ is the scaled gradient of the void fraction in the direction $(u=1, r=0)$, so along $r=g(u)$, or $c_{\mathrm{L}}=k(u)$ (see Fig. 1), i.e., the composition at minimum void fraction. This constitutes the maximum gradient, which is pertaining to the distribution $\alpha=0$ (see Sec. III). Equation (49) also expresses that the gradient of the void fraction in the direction of $r$, i.e., $d h / d r(u=1, r=1)$, is zero (corresponding to the direction of the variable $c_{\mathrm{L}}$ in Fig. 1). This feature of the gradients in void fraction holds for all bimodal particle packings, and confirms that for all continuous PSD the maximum packing fraction is obtained for a power law distribution having $\alpha=0$.

Substituting Eqs. (48) and (50) and into Eq. (47) yields the void fraction of a continuous power law packing

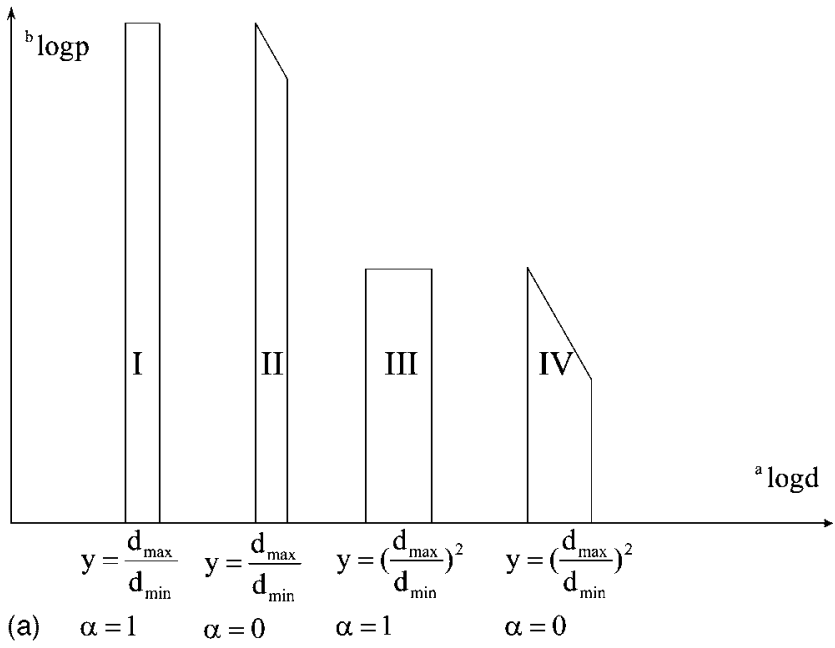

(b)

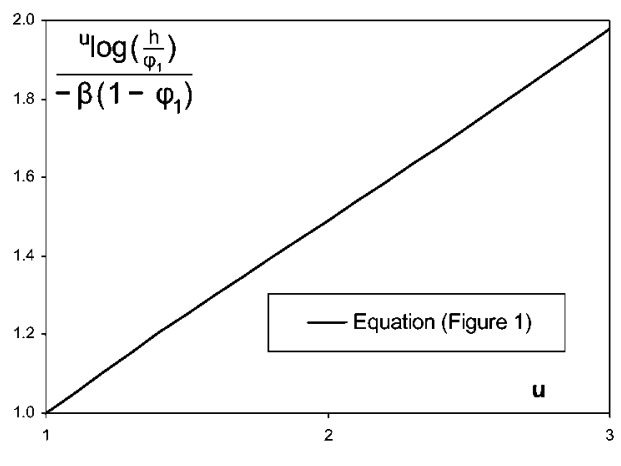

FIG. 7. (a) Four continuous particle-size distributions whereby the size width $\left(y\right.$ and $\left.y^{2}\right)$ and distribution modulus $\alpha(0$ and 1) are varied. (b) Scaled exponent of Eq. (52) versus size width $u$ at composition of maximum RLP of spheres $[h(u, r=g(u)]$, invoking the values $\left(\varphi_{1}=0.5, \beta=0.125\right)$ and expressions used in Fig. 1 . The values of $\alpha$ pertaining to $u$ can be found in Fig 2(b) The limit $u=1$ corresponds to a continuous distribution for which $\alpha=0$.

$$
\begin{aligned}
\varphi & =\lim _{n \rightarrow \infty}\left[1-\frac{\beta\left(1-\varphi_{1}\right)}{n\left(1+\alpha^{2}\right)} \ln y+\mathrm{O}\left(\frac{1}{n^{2}}\right)\right]^{n-1} \\
& =\varphi_{1} y^{-\left(1-\varphi_{1}\right) \beta /\left(1+\alpha^{2}\right)}=\varphi_{1}\left(\frac{d_{\max }}{d_{\min }}\right)^{-\left(1-\varphi_{1}\right) \beta /\left(1+\alpha^{2}\right)} .
\end{aligned}
$$

Equation (51) provides the void fraction of a continuous power low PSD [governed by Eqs. (34) or (35)], which depends on the distribution width $(y)$, the exponent of the particle distribution shape $(\alpha)$, the void fraction of the singlesized particles $\left(\varphi_{1}\right)$ and the maximum gradient of the singlesized void fraction on the onset to bimodal packing $(\beta)$. Equation (51) indicates that the void fraction of the system tends to the monosized void fraction when the distribution width tends to unity, and/or when $\alpha$ tends to $-\infty$ or $\infty$ (i.e., the distribution tending to uniformly sized distribution of sizes $d_{\min }$ or $d_{\max }$, respectively), as would be expected. Equation (51) also reveals the effect of distribution modulus and size width on void fraction. To this end, in Fig. 7(a), four different distributions are given. From Eq. (51), it readily follows that the exponent of $y$ [appearing in Eq. (51)] of 
TABLE IV. Experimental data [A, B, $C$ values, Ref. [34] and Eq. (53)] and values derived therefrom, experimental data from Refs. [24,18]; values assessed in this study; data derived from computer simulations (Ref. [10]) and based on an expression given by Refs. [6,7,33] for binary fcc packing: $h=1-\left(1-\varphi_{1}\right)[1$ $\left.-c_{\mathrm{S}}\left(1-u^{-3}\right)\right]$.

\begin{tabular}{lcccccccc}
\hline \hline \multicolumn{1}{c}{ Material } & Packing & Shape & $A$ & $B$ & $C$ & $\varphi_{1}$ & $\beta$ & $\beta\left(1-\varphi_{1}\right)$ \\
\hline Steel $^{\mathrm{a}}$ & $\mathrm{RCP}$ & spherical & - & - & - & 0.375 & 0.140 & 0.0875 \\
Simulation $^{\mathrm{b}}$ & $\mathrm{RCP}$ & spherical & - & - & - & 0.360 & 0.203 & 0.0973 \\
Steel $^{\mathrm{c}}$ & $\mathrm{RLP}$ & spherical & - & - & & 0.500 & 0.125 & 0.0625 \\
Equation $^{\mathrm{d}}$ & $\mathrm{fcc}$ & spherical & - & - & - & 0.260 & -5.780 & -4.280 \\
Quartz $_{\text {Feldspar }}$ & $\mathrm{RCP}$ & fairly angular & 0.503 & 0.731 & 0.374 & 0.497 & 0.374 & 0.1881 \\
Dolomite $_{\text {Sillimanite }}$ & $\mathrm{RCP}$ & plate-shaped & 0.497 & 0.722 & 0.374 & 0.503 & 0.374 & 0.1859 \\
Flint & $\mathrm{RCP}$ & fairly rounded & 0.495 & 0.700 & 0.347 & 0.505 & 0.347 & 0.1718 \\
\hline \hline
\end{tabular}

${ }^{\mathrm{a}}$ Experimental data from Ref. [24].

${ }^{\mathrm{b}}$ Data derived from computer simulations in Ref. [10].

${ }^{c}$ Experimental data from Ref. [18].

${ }^{\mathrm{d}}$ Data based on expressions given in Refs. [6,7,33].

${ }^{\mathrm{e}}$ Values assessed in this study, based on data from Ref. [20].

packings I, II, III, and IV of Fig. 7(a) have mutual ratios $4: 2: 2: 1$, respectively. By measuring the packing density of fractions with various size widths, Eq.(51) also enables the derivation of the true monosized packing fraction of an irregularly shaped particle.

Furthermore, the derivation presented here also permits the comparison of the packing fraction, for a given size width $y$, versus the size ratio of the discrete distribution. To this end, Eqs. (8) and (47) are combined to

$$
\varphi=\varphi_{1} y^{u^{\log \left(h(u, r) / \varphi_{1}\right)} .}
$$

Using the expression for $h$ and $r[=g(u)]$ given in Fig. 1, one can compute the exponent of Eq. (52) for the densest RLP of spheres as a function of the size ratio $u$. In Fig. 7(b) the scaled exponent is set out. One can, for instance, see that compared to the continuous distribution $(u=1, \alpha=0)$, the discrete distribution with $u=2(\alpha=0.11)$ has an exponent that is about a factor of 1.5 larger, i.e., a larger reduction in void fraction is achieved using discretely sized spheres. The maximum geometric packing is obtained with saturated discrete distributions. For RLP of spheres $u=u_{b} \approx 10, h \approx \varphi_{1}^{2}$, the exponent of Eq. (52) yields -0.30 , and the scaled exponent featuring in Fig. 7(b) then would attain a value of 4.8. In this case the exponent of Eq. (52) corresponds to $-\alpha$, see Eq. (43), $\alpha$ being the exponent of the cumulative distribution function.

\section{Void fraction gradient of unimodal/bimodal discrete packing}

In Eq. (51) the gradient of the single-sized void fraction on the onset to bimodal packing $(\beta)$ features. For the RLP of spheres the experimental data of Furnas [18] fitting yields $\beta=0.125$ [based on data Tables I and II], whereas for the RCP of spheres the experimental data of McGeary [24] the best fit yields $\beta=0.14$ [Table III]. The RCP packing/void fraction gradient can also be derived from computergenerated packings. Kansal et al. [10] computed the RCP of bidisperse spheres in the range $1 \leqslant u \leqslant 10$. In the vicinity of $u=1$ and $\varphi_{1}=0.64$, from their Fig. 5 it follows that $\Delta u=0.71\left(u^{3}=5\right)$ and $\Delta h=0.025$, and considering Eq. (49), that the scaled gradient is about 0.152 . This is the gradient pertaining to $c_{\mathrm{L}}=0.75$ and $c_{\mathrm{S}}=0.25$. As $\beta$, the maximum gradient, is found and defined at $c_{\mathrm{L}}=c_{\mathrm{S}}=0.5$ and the void fraction gradient is proportional to $c_{\mathrm{L}} c_{\mathrm{S}}$, it follows that $\beta=0.203$. So, both the packing fraction $f_{1}$ and the gradient $\beta$ are larger following the numerical simulation [10] than following the experiments [24]. In Table IV, $\varphi_{1}$ and $\beta$ of both RLP and RCP of spheres are included.

Also for other packings (binary fcc packings and random irregular particle packings) information on the void fraction in the vicinity of $u=1$ is available. From the expression used in Refs. [6,7,33], it follows that for fcc, $\beta=-3 / 2\left(1-f_{1}\right)\left(\approx-5.78\right.$ as $\left.f_{1}=2^{1 / 2} \pi / 6\right)$. The negative value reflects the reduced packing of the lattice at the onset from a monosphere lattice to a slightly disordered bimodal lattice. This is in contract to random packings, where a contraction (packing fraction increase) occurs when spheres of two different sizes are combined.

For randomly packed irregularly shaped particles, only experimental data is available. Patankar and Mandal [34] determined the minimum of the vibrated bimodal void fraction versus the size ratio, and obtained the same trend as Fig. 5. A line of the form

$$
H(u)=\frac{1-\varphi_{1}-A+B e^{-C u}}{1-\varphi_{1}},
$$

was fitted, and in Table IV their fitted $A, B$, and $C$ are summarized. Note that $A=B e^{-C}$ since $H(u=1)=1$, that $1-A=\varphi_{1}$ for $H(u \rightarrow \infty)=0$, and that $C=\beta$ [in view of Eq. (49)]. In Table IV also the values of $\varphi_{1}$ and $\beta$ are summa- 
TABLE V. Fractions of broken flint created and used in Ref. [20] to compose the continuous power law distributions and to measure the void fraction of these packings (as depicted in Fig. 8).

\begin{tabular}{cc}
\hline \hline Sieve No. & $d_{i}(\mathrm{~mm})$ \\
\hline 1 & 3.00 \\
2 & 2.00 \\
3 & 1.25 \\
4 & 0.78 \\
5 & 0.53 \\
6 & 0.41 \\
7 & 0.245 \\
8 & 0.150 \\
9 & 0.105 \\
10 & 0.065 \\
11 & 0.05 \\
12 & 0.04 \\
13 & 0.025 \\
\hline
\end{tabular}

rized. Compared to close spherical particle packing $\left(\varphi_{1}\right.$ $=0.375$ ), the void fraction of their (also monosized) close irregular particles appears to be higher $\left(\varphi_{1} \approx 0.5\right.$, Table IV). But upon grading, the latter appear to exhibit a larger reduction in void fraction as $\beta$ is typically 0.39 , i.e., about two times the value as found for the spheres; see the discussion above.

The binary packing experiments $[18,24,34]$ and numerical simulations [10] all indicate that for random packings the derivative of the packing fraction with respect to the size ratio $u$ is positive at $u=1$. As this increase is fairly linear in $u$ and symmetrical with respect to $c_{\mathrm{L}}=c_{\mathrm{S}}=0.5$ (Fig. 1), it can adequately be approximated to be proportional to $(u-1) c_{\mathrm{L}} c_{\mathrm{S}}$. As a direct consequence, the scaled gradient $\beta$ is nonzero as well, and the power law packing is predicted correctly to be larger than the monosized packing [Eqs. (49) and (51)]. Packing models based on the Percus-Yevick (PY) equation, on the other hand, yield a system contraction proportional to $(u-1)^{2} c_{\mathrm{L}} c_{\mathrm{S}}$, e.g., see Ref. [3]. The gradient of the packing fraction is then predicted to be zero at $u=1$, which is questionable. This PY equation originates from the compressibility theory of fluids, and seems to be applicable to model hard sphere systems only when the packing density is not close to its maximum.

\section{Experimental validation}

A thorough verification of Eq. (51) is possible by comparing it with the grading and packing fraction experiments by Andreasen and Andersen [20]. They sieved broken flint on ten sieves (Table V), and the $7 \%$ lying on the largest sieve (No. 1) with size $3 \mathrm{~mm}$ was discarded. The fraction passing the smallest sieve (No. 10), was further separated in three fractions passing (most likely) $0.05 \mathrm{~mm}, 0.04 \mathrm{~mm}$, and $0.025 \mathrm{~mm}$, which are added as "sieves" No. 11 to No. 13 in Table V. With these 13 fractions they composed continuous

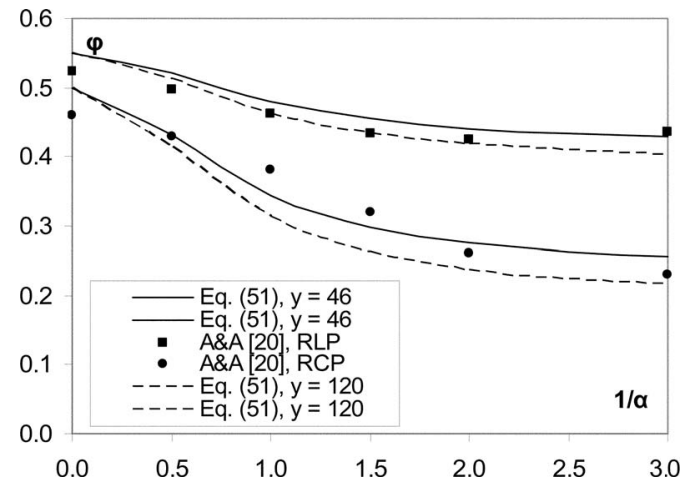

FIG. 8. Experimentally measured void fractions of continuously graded packings as given in Fig. 9 of Ref. [20] and theoretical prediction using Eq. (51).

power law particle-size distributions (Fig. 7 in Ref. [20]), with $d_{\max }=3 \mathrm{~mm}$ and various $d_{\min }$ and $\alpha$, that follow Eq. (12), and the void fractions of their packings were measured (Fig. 9 in Ref. [20]).

From Fig. 7 of Ref. [20] and Table V, one can derive that that the size ratio of their composed packings, $y=d_{\max } / d_{\min }$, amounted $46\left(d_{\min }=0.065 \mathrm{~mm}\right.$ for $\alpha=1$ and 2$)$, $75\left(d_{\min }=0.04 \mathrm{~mm}\right.$ for $\alpha=1 / 2$ and $\left.2 / 3\right)$ to 120 $\left(d_{\min }=0.025 \mathrm{~mm}\right.$ for $\left.\alpha=1 / 3\right)$. The unimodal void fraction ( $\alpha^{-1}=0$ in Fig. 9 of Ref. [20]), they assigned to the void fraction of the material between sieves No. 9 and No. 10, which have a size ratio of 1.5. Accordingly, their "monosized" void fraction of loose and close packings amount to 0.52 and 0.46 , respectively. Their closely packed void fraction is less than the values measured by Patankar and Mandal [34], $\varphi_{1} \approx 0.50$ see Table IV. Patankar and Mandal [34] employed a size ratio of about 1.2 to assess the monosized void fraction, resulting in higher monosized void fraction, which is closer to reality. Substituting as lower and upper $y$ values 46 and 120, respectively, $\varphi_{1}=0.50$ (Table IV), $\beta=0.39$ (Table IV), $\varphi$ is computed with Eq. (51) for various $\alpha$ and included in Fig. 8, in which also measured values of Ref. [20] appear. One can see that Eq. (51), derived here for the first time, provides a good prediction of the void fraction versus the reciprocal distribution modulus. To apply this analytically exact result for the void fraction, one only needs the bimodal data (here from Ref. [34]), and no additional fitting parameters are needed.

For the loose packing of irregular particles, bimodal values of $\varphi_{1}$ and $\beta$ are, to the author's knowledge, not yet available. Accordingly, based on the value provided by Ref. [20], being underestimation $\varphi_{1}=0.52, \varphi_{1}=0.55$ is taken as loose monosized void fraction of broken material, and $\beta=0.16$ is obtained by fitting. This value of the gradient in unimodal/ bimodal void fraction of loose angular material is a little greater than the corresponding value (loose, bimodal) of sphere packing fraction as measured in Ref. [18]. Hence, it appears that both this coefficient, which constitutes the gradient of void reduction when a unimodal packing becomes a bimodal packing, and the monosized packing fraction depend less on particle shape for loose packings (in contrast to close packings). 
The extensive comparison of Eq. (51) with the results of Ref. [20] results in good agreement for variable $\alpha$, but concerned values of $y$ in a limited range (46 to 120) only. However, Eq. (51) appears also to be in line with classical work by Caquot [23], who measured the voids of granulate mixes of cement, sand, and gravel that had distribution widths $(y)$ up to several thousands. Based on numerous experiments, the following empirical formula was proposed:

$$
\varphi=0.35\left(d_{\max } / 1 \mathrm{~mm}\right)^{0.2} .
$$

A glance at Eq. (51), considering that $\beta\left(1-\varphi_{1}\right) \approx 0.18$ (Table IV) and that for the Caquot packings holds $\alpha=0.20$ (Sec. III), so that $1+\alpha^{2}=1.04$, confirms the compatibility of empirical equation (54) and the theoretically derived Eq. (51). Dated Eq. (54), which was almost fallen into oblivion, is also discussed in Ref. [35].

Hummel [36] investigated the packing fraction of composed continuous power law packings for $\alpha$ ranging from 0.05 to unity, using natural river aggregates (sand and gravel) and broken basalt. A $u=2$ sieve set with $d_{\min }=0.2 \mathrm{~mm}$ and $d_{\max }=30 \mathrm{~mm}$ was employed to classify the materials, and Eq. (12) composed. For the more spherically aggregates maximum packing was found for $\alpha \approx 0.37$ (RCP and RLP), and for the angular basalt particles this $\alpha \approx 0.28$ (RCP and $\mathrm{RCP})$.

In this section the packing fraction of geometric packings has been analyzed. The present analysis and the experimental findings of Furnas, Anderegg, Andreasen and Andersen, Caquot, and Hummel, in essence result in the same ideal grading line to achieve a maximum packing fraction and minimum void fraction of geometric packings [Eqs. (30), (34), and (35) or (37)]. The $\alpha$ for maximum packing depends on the size ratio $u$ and on the concentration ratio $r$ of the sizes, Eq. (31). For packings composed of sieve classes, Eq. (30) results in the highest packing fraction, both $r(u)>1$ and $\alpha$ $={ }^{u} \log r>0$, typically $\alpha=0.20-0.37$ for $u$ of $\sqrt{2}$ to 2 (Refs. $[19,23,31,36])$. Completely controlled populations ("infinite number of sieves"), so $u \rightarrow 1$, the continuous distribution should obey Eq. (35) as in the limit $u \rightarrow 1, \alpha=0$ provides densest packing fraction. This result follows among others from studying the transition from unimodal to bimodal packing (Fig. 1) and prevailing gradients in void fraction [Eq. (49)], and from experimental [25] and simulation [26] work.

The packing/void fraction of continuous power law packings in essence depends on the single-sized void fraction, the distribution modulus, and the magnitude of the size range, i.e., $y\left(=d_{\max } / d_{\min }\right)$. The uniform void fraction in turn will depend on the particle shape and packing mode (e.g., loose, close). It appears that Eq. (51), derived for continuous power law distributions, is also suited for describing the void fraction of continuous quasi power law packings composed of sieved material for a wide range of $\alpha$ (Fig. 8).

\section{CONCLUSIONS}

In the present paper, the particle-size distribution and void fraction of geometric random packings, consisting of equally shaped particles, are addressed. It is demonstrated that the void fraction of a bimodal discrete packing in the limit of the size ratio $d_{\mathrm{L}} / d_{\mathrm{S}}$ tending to unity (so, towards the unimodal packing), contains important information in regard to the discrete [Eqs. (30) and (37)] and continuous [Eqs. (34) and (35)] geometric distributions. The gradient in void fraction ranges from 0 to $\beta\left(1-\varphi_{1}\right) \varphi_{1}$, and depends on the angle $\gamma$ (Fig. 6), which is directly related to the distribution modulus $\alpha$ of the power law distribution.

The values of $\varphi_{1}$ and $\beta$ are extracted from the experimental and simulation data in discrete bimodal packings $[10,18,24,34]$ and are summarized in Table IV. Likewise for the unimodal void fraction $\varphi_{1}$, also $\beta$ depends only on the particle shape and the method of packing. For the close packing the $\beta$ values of spheres and irregular particles differ significantly, whereas this difference in $\beta$ is smaller when the particles are packed in loose state (as is also the case for the monosized packing fraction). The opposite signs of $\beta$ for a random packing and a fcc lattice reflect the contraction and expansion of these packings, respectively, when the monosized packing becomes bimodal.

The present analysis also addresses the maximum packing fraction of multiple discrete particles as function of the size ratio (whereby the limit $u=1$ implies a continuous geometric distribution). Based on the RLP of spheres data from Furnas [18], Fig. 2(b) provides the exponent $\alpha$, which is positive for $u>1$, of Eq. (30) for a maximum packing fraction. Subsequently, Fig. 7(b) reveals the possible reduction in packing fraction as a function of the size ratio (for each size ratio this optimum $\alpha$ used).

It follows that for continuous (power law) distributions of particles a maximum packing fraction is obtained for $\alpha=0$. The void fraction of a power law packing with arbitrary values of $\alpha$ follows from basic Eq. (51). In general, the void fraction reduction by correct grading is more pronounced when the monosized void fraction $\varphi_{1}$ is lower, and $\beta$ is larger, as is the case with close packing of irregular particles (e.g., sand, cement). This void fraction prediction is furthermore found to be in good quantitative agreement with the classical experiments [20], as is illustrated by Fig. 8, and with the empirical relation [Eq. (54)] given by Caquot [23].

In the past, various researchers have tried to obtain the densest packing fraction of continuously graded systems using sieved fractions and composing quasi continuous geometric packings of them (e.g., Refs. [19,31]), for which Eq. (30) appears to be valid too. They all recommended a constant ratio $(r)$ between the amounts of material on consecutive screens sizes of constant size ratio $(u)$, i.e., forming a geometric progression similar to the one composed from discretely sized particles. The unification of discrete and continuous particle packings as presented here, also enables the coupling of the exponent $\alpha$ of the discrete power law distribution to these "sieve laws;" Eq. (31). Analyzing the data from Refs. [19,23,31,36] which used consecutive screens of constant size ratios $\sqrt{2}$ or 2 and various particle shapes, yields $\alpha=0.20$ to 0.37 to obtain the densest packing fraction. These positive values of $\alpha$ are due to the fact that the population of each sieve class cannot be controlled, even when the employed sieve size ratio $u$ is $\sqrt{2}$. In the limit of $u$ tending to unity, viz. composing a perfect continuous power law distribution, $\alpha=0$ as discussed above will yield a maximum packing fraction. 


\section{ACKNOWLEDGMENTS}

The author wishes to thank G. Hüsken for his assistance with the drawing of the figures. The author also would like to express his gratitude towards the following persons and institutions for providing copies of references: C. Field from the Portland Cement Association (PCA), Skokie, Illinois, U.S., M. Wollschläger from the Verein Deutscher Zementwerke e.V. (VDZ), Düsseldorf, Germany, and C. Baudet from the Laboratoire des Ecoulements Géophysiques et Industriels, Grenoble, France.
[1] S. H. Liou and C. L. Chien, Phys. Rev. B 35, 2443 (1987).

[2] O. N. Senkov and D. B. Miracle, Mater. Res. Bull. 36, 2183 (2001).

[3] J. L. Lebowitz and J. S. Rowlinson, J. Chem. Phys. 41, 133 (1964).

[4] N. F. Carnahan and K. E. Starling, J. Chem. Phys. 51, 635 (1969).

[5] H. J. Hermann, R. Mahmoodi Baram, and M. Wackenhut, Physica A 330, 77 (2003).

[6] A. R. Denton and N. W. Ashcroft, Phys. Rev. A 42, 7312 (1990).

[7] P. Jalali and M. Li, Phys. Rev. B 71, 014206 (2005).

[8] R. Mahmoodi Baram, H. J. Hermann, and N. Rivier, Phys. Rev. Lett. 92, 044301 (2004).

[9] G. D. Scott, Nature (London) 188, 908 (1960); G. D. Scott and D. M. Kilgour, Br. J. Appl. Phys., J. Phys. D 2, 863 (1969).

[10] A. R. Kansal, S. Torquato, and F. H. Stillinger, J. Chem. Phys. 117, 8212 (2002).

[11] A. R. Kansal, S. Torquato, and F. H. Stillinger, Phys. Rev. E 66, 041109 (2002).

[12] G. Y. Onoda and E. G. Liniger, Phys. Rev. Lett. 64, 2727 (1990).

[13] J. G. Berryman, Phys. Rev. A 27, 1053 (1983).

[14] A. P. Philipse, Langmuir 12, 1127 (1996).

[15] W. Man, A. Donev, F. H. Stillinger, M. T. Sullivan, W. B. Russel, D. Heeger, S. Inati, S. Torquato, and P. M. Chaikin, Phys. Rev. Lett. 94, 198001 (2005).

[16] H. Y. Sohn and C. Moreland, Can. J. Chem. Eng. 46, 162 (1968).

[17] D. He, N. N. Ekere, and L. Cai, Phys. Rev. E 60, 7098 (1999).

[18] C. C. Furnas, Department of Commerce, Bureau of Mines, Report of Investigation Serial No. 2894, 1928; Bulletin of US Bureau of Mines 307, 74 (1929).

[19] C. C. Furnas, Ind. Eng. Chem. 23, 1052 (1931).

[20] A. H. M. Andreasen and J. Andersen, Kolloid-Z. 50, 217 (in
German) (1930).

[21] J. Zheng, P. F. Johnson, and J. S. Reed, J. Am. Ceram. Soc. 73, 1392 (1990).

[22] J. E. Funk and D. R. Dinger, Predictive Process Control of Crowded Particulate Suspension, Applied to Ceramic Manufacturing (Kluwer Academic Press, Boston, 1994).

[23] M. A. Caquot, Mémoires de la Société des Ingénieurs Civils de France, 562 (in French) (1937).

[24] R. K. McGeary, J. Am. Chem. Soc. 44, 513 (1961).

[25] P. C. Mangelsdorf and E. L. Washington, Nature (London) 187, 930 (1960).

[26] A. S. Clarke and J. D. Wiley, Phys. Rev. B 35, 7350 (1987).

[27] O. Redlich and A. T. Kister, J. Am. Chem. Soc. 71, 505 (1949).

[28] Also along $\left(u \geqslant 1, c_{\mathrm{L}}=0\right)$ and $\left(u \geqslant 1, c_{\mathrm{L}}=1\right)$ the void fraction remains $\varphi_{1}$, as this corresponds to the packing of unimodal spheres with diameter $d_{\mathrm{S}}$ and $d_{\mathrm{L}}$, respectively.

[29] H. J. H. Brouwers, University of Twente Report No. 2005R003/CME-001, 2005 (unpublished).

[30] Often continuous populations (sieve classes) are characterized by one discrete size, e. g., by the geometric mean $\left(\sqrt{d_{i} d_{i+1}}\right)$ or by the arithmetic mean $\left(1 / 2 d_{i}+1 / 2 d_{i+1}\right)$ of a class. In each of these cases, the resulting discrete size ratio amounts $\mathrm{u}$ as well as $d_{i+1}=d_{i} u$.

[31] F. O. Anderegg, Ind. Eng. Chem. 23, 1058 (1931).

[32] A. M. Gaudin, Petroleum Development and Technology 73, 253 (1926).

[33] A. R. Denton (private communication).

[34] A. N. Patankar and G. Mandal, Trans. J. Br. Ceram. Soc. 79, 59 (1980).

[35] J. Baron and R. Sauterey, Le béton hydraulique, connaissance et pratique (in French) (Presses de l'Ecole Nationale des Ponts et Chaussées, Paris, 1982).

[36] A. Hummel, Das Beton-ABC, Ein Lehrbuch der Technologie des Schwerbetons und des Leichtbetons (in German) (Verlag von Wilhelm Ernst \& Sohn, Berlin, 1959). 
PHYSICAL REVIEW E 74, 069901(E) (2006)

\title{
Erratum: Particle-size distribution and packing fraction of geometric random packings [Phys. Rev. E 74, 031309 (2006)]
}

\author{
H. J. H. Brouwers
}

(Received 30 October 2006; published 12 December 2006)

DOI: 10.1103/PhysRevE.74.069901

PACS number(s): 45.70. $-\mathrm{n}, 81.05 . \mathrm{Rm}, 99.10 .-\mathrm{x}$

In this paper two typographical errors have occurred. On p. 11, right column, 5 th line, 0.025 should read -0.025 . On p. 13, left column, 2nd paragraph, last line, "RCP and RCP" should read "RCP and RLP."

Both typographical errors have no further impact on the content of the paper. 